\title{
To what extent does emotional dysregulation account for aggression associated with ADHD symptoms? An experience sampling study
}

\author{
Murray, Aja ; Lavoie, Jennifer ; Booth, Tom ; Eisner, Manuel ; Ribeaud, Denis
}

\begin{abstract}
Previous research suggests that aggression is associated with ADHD symptoms and this may partly reflect problems with emotional regulation. Using data from the D2M study $(n=260)$ we found that ADHD symptoms were associated with both emotional lability and aggression, but emotional lability did not mediate the ADHD-aggression association. Results suggest that other factors may be more important for explaining elevated levels of aggression in ADHD.
\end{abstract}

DOI: https://doi.org/10.1016/j.psychres.2021.114059

Posted at the Zurich Open Repository and Archive, University of Zurich

ZORA URL: https://doi.org/10.5167/uzh-212987

Journal Article

Accepted Version

Originally published at:

Murray, Aja; Lavoie, Jennifer; Booth, Tom; Eisner, Manuel; Ribeaud, Denis (2021). To what extent does emotional dysregulation account for aggression associated with ADHD symptoms? An experience sampling study. Psychiatry Research, 303:114059.

DOI: https://doi.org/10.1016/j.psychres.2021.114059 
To what extent does emotional dysregulation account for aggression associated with ADHD symptoms? An experience sampling study

Aja Murray ${ }^{1}$, Jennifer Lavoie ${ }^{2}$, Tom Booth ${ }^{1}$, Manuel Eisner ${ }^{2,3}$, Denis Ribeaud ${ }^{3}$

${ }^{1}$ Department of Psychology, University of Edinburgh

${ }^{2}$ Institute of Criminology, University of Cambridge

${ }^{3}$ Jacobs Center for Productive Youth Development, University of Zurich 


\begin{abstract}
Previous research suggests that aggression is associated with ADHD symptoms and this may partly reflect problems with emotional regulation. Using data from the D2M study ( $\mathrm{n}=260)$ we found that ADHD symptoms were associated with both emotional lability and aggression, but emotional lability did not mediate the ADHD-aggression association. Results suggest that other factors may be more important for explaining elevated levels of aggression in ADHD.
\end{abstract}

Keywords: ADHD; aggression; emotional dysregulation; experience sampling; ecological momentary assessment 
Aggression commonly occurs with ADHD symptoms, where it represents a major source of impairment (e.g., see Saylor \& Amann, 2016 for a review). Emotional dysregulation (also commonly associated with ADHD symptoms) has been proposed as a potential mediating mechanism. Emotional dysregulation can be conceptualised as having multiple facets (see Faraone et al., 2019 for a review); however, Slaughter et al. (2019) proposed that the lability of negative emotions may be a particularly relevant for explaining the link between ADHD and aggression. Negative emotional lability can be defined as the tendency to experience sharp, intense shifts of negative emotion (e.g., anger, sadness, fear) that are not commensurate with situation or developmental stage. Though useful questionnaire measures of emotional lability have been developed, negative emotional lability is arguably best operationalised using experience sampling designs that can capture changes in emotions from moment-to-moment in the course of daily life (e.g., Factor et al., 2014). As they are completed during or shortly after a relevant event (e.g., an affective state), experience sampling measures of emotional lability have greater ecological validity and can help overcome issues such as retrospective recall bias that affect questionnaire measures (Rosen \& Factor, 2015). Thus, studies that have used experience sampling designs are especially valuable in assessing a mediating role of emotional dysregulation in the association between ADHD symptoms and aggression (e.g., Rosen \& Factor, 2015; Slaughter et al., 2019).

We employed an experience sampling-based methodology to evaluate the mediating role of emotional dysregulation, specifically, emotional lability in the co-occurrence of ADHD symptoms and aggression. To avoid the potential inflation of ADHD-aggression-emotional dysregulation cooccurrence estimates due to Berkson's bias i.e., the over-estimation of the extent of symptom cooccurrence in clinically ascertained samples and reflecting the fact that both ADHD symptoms and aggression vary meaningfully both above and below clinical cut-offs, we used a communityascertained rather than a clinical sample.

\section{Methods}

\section{Ethics}


Ethical approval was obtained from the Ethics Committee from the Faculty of Arts and Social Sciences of the University of Zurich (ECFASSUZH). Participants provided informed consent before participating.

\section{Participants}

Participants were from the D2M study, the procedure for which is described in Supplementary Materials and previous publications (Murray et al., 2020). Participants (n=260; 100 males; median age 20) represented a convenience sample from the normative z-proso longitudinal study. The only inclusion criterion was that participants were required to have access to a smartphone running iOS or android operating systems in order to be able to download the smartphone application through which the experience sampling survey was administered. The D2M sample do not appear to differ from the main cohort on their levels of ADHD symptoms $(t(434.9)=1.20, p=.17)$; however, they score slightly lower on aggression $(t(516.7)=-2.92, p=.004)$. The D2M sample also has a higher proportion of females than the main cohort (which has an approximately equal gender split).

\section{Measures}

ADHD symptoms and aggression were measured using an adapted self-reported version of the Social Behavior Questionnaire, administered as part of the age 20 main wave of z-proso and emotional lability was based on mean squared successive differences calculated from the negative emotion item scores of the PANAS-X. Further information on the measures is provided in Supplementary Materials.

\section{Analysis}

The structural equation model summarised in Figure 1 was fit to test the mediating role of a latent emotional lability factor in the association between a latent ADHD symptoms factor and latent aggression factors. The statistical significance of the indirect effects was assessed based on bootstrapped standard errors, using 1000 bootstrapped samples. Full details are provided in Supplementary Materials. Models were fit using lavaan in R statistical software using full information maximum likelihood estimation (FIML). 


\section{Results}

Results are summarised in Figure 1 and suggested that while there was a significant effect of ADHD symptoms on both emotional lability and all four types of aggression assessed, there was no significant effect of emotional lability on any type of aggression. There were also no significant indirect effects of ADHD symptoms on any type of aggression via emotional lability: the unstandardized parameters for the indirect effects were for reactive aggression: $b=0.05(95 \% \mathrm{CI}=-$ 0.02 to 0.17$)$, for indirect aggression: $b=-0.01(95 \% \mathrm{CI}=-0.07$ to 0.06$)$, for proactive aggression: $b=$ $0.00(95 \% \mathrm{CI}=-0.04$ to 0.06$)$, and for physical aggression: $\mathrm{b}=-0.01(95 \% \mathrm{CI}=-0.05$ to 0.03$)$.

\section{Discussion}

Aggression commonly co-occurs with ADHD symptoms. It has previously been proposed that negative affective lability may partly account for the elevated rates of aggression observed in individuals with ADHD symptoms (Slaughter et al., 2019). Using measures of emotional lability based on real life emotional functioning we found that ADHD symptoms had a significant effect on emotional lability as well as reactive, proactive, indirect, and physical aggression. However, emotional lability did not mediate the association between ADHD symptoms and aggression.

Our study replicates others that have identified a link between ADHD symptoms and aggression on the one hand (Murray et al., 2016) and ADHD symptoms and emotional dysregulation on the other (Faraone et al., 2019). It also adds to the small but growing literature demonstrating associations between ADHD symptoms and manifestations of emotional dysregulation in the context of daily life, captured through experience sampling methodologies (Miguelez-Fernandez et al., 2018). However, only a handful of studies have simultaneously examined ADHD symptoms, aggression, and 'real life' emotional dysregulation (Rosen et al., 2015; Rosen \& Factor, 2015; Slaughter et al., 2019) and none have directly evaluated whether the latter mediates the association between the former two constructs. Our results thus add important evidence that while ADHD symptoms are related to both negative emotional lability and aggression, the former association may not explain the latter; mechanisms other than those reflected by negative affective lability reflects likely account for the 
ADHD-aggression association. Some possible explanations, limitations and future directions are discussed in Supplementary Materials.

\section{Conclusions}

Our results confirm that ADHD symptoms are associated with both emotional dysregulation and reactive, proactive, indirect and physical aggression. Emotional dysregulation did not, however, mediate the association between ADHD symptoms and aggression, suggesting that the association is due to other mechanisms. 


\section{References}

Factor, P. I., Reyes, R. A., \& Rosen, P. J. (2014). Emotional impulsivity in children with ADHD associated with comorbid-Not ADHD—symptomatology. Journal of Psychopathology and Behavioral Assessment, 36(4), 530-541.

Faraone, S. V., Rostain, A. L., Blader, J., Busch, B., Childress, A. C., Connor, D. F., \& Newcorn, J. H. (2019). Practitioner Review: Emotional dysregulation in attention-deficit/hyperactivity disorder-implications for clinical recognition and intervention. Journal of Child Psychology and Psychiatry, 60(2), 133-150.

Miguelez-Fernandez, C., de Leon, S. J., Baltasar-Tello, I., Peñuelas-Calvo, I., Barrigon, M. L., Capdevila, A. S., Delgado-Gómez, D., Baca-García, E., \& Carballo, J. J. (2018). Evaluating attention-deficit/hyperactivity disorder using ecological momentary assessment: A systematic review. ADHD Attention Deficit and Hyperactivity Disorders, 10(4), 247-265.

Murray, A. L., Obsuth, I., Zirk-Sadowski, J., Ribeaud, D., \& Eisner, M. (2016). Developmental relations between ADHD symptoms and reactive versus proactive aggression across childhood and adolescence. Journal of Attention Disorders, 1087054716666323.

Murray, A. L., Wong, S.-C., Obsuth, I., Rhodes, S., Eisner, M., \& Ribeaud, D. (2020). An ecological momentary assessment study of the role of emotional dysregulation in co-occurring ADHD and internalising symptoms in adulthood. Journal of Affective Disorders. In press.

Rosen, P. J., \& Factor, P. I. (2015). Emotional impulsivity and emotional and behavioral difficulties among children with ADHD: An ecological momentary assessment study. Journal of Attention Disorders, 19(9), 779-793.

Rosen, P. J., Walerius, D. M., Fogleman, N. D., \& Factor, P. I. (2015). The association of emotional lability and emotional and behavioral difficulties among children with and without ADHD. ADHD Attention Deficit and Hyperactivity Disorders, 7(4), 281-294. 
Saylor, K. E., \& Amann, B. H. (2016). Impulsive aggression as a comorbidity of attentiondeficit/hyperactivity disorder in children and adolescents. Journal of Child and Adolescent Psychopharmacology, 26(1), 19-25.

Slaughter, K. E., Leaberry, K. D., Fogleman, N. D., \& Rosen, P. J. (2019). Reactive and proactive aggression in children with and without ADHD and negative emotional lability. Social Development. 
Figure 1: Standardised structural parameters for the structural equation model examining the direct and indirect effects of ADHD symptoms on aggression

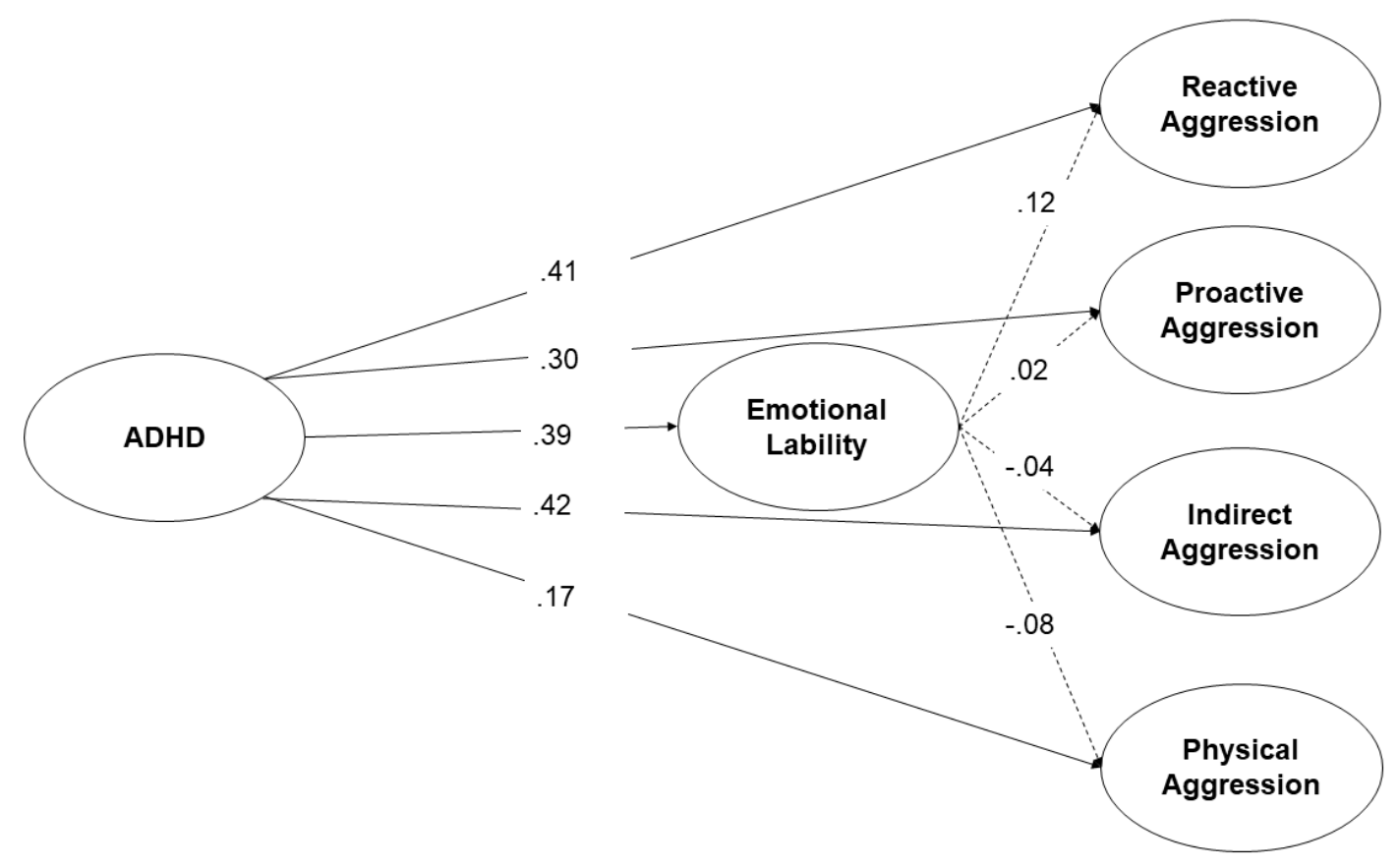

Note. Measurement models and residual covariances between aggression factors omitted for visual clarity. Solid lines represent statistically significant paths $(p<.05)$ and dashed lines represent nonsignificant paths. 